those brought by Internet use and smart phones, before moving on to spatial and territorial planning. That word territory crops up again, and it is almost as if we were back in the first section as we look at very similar maps. The difference is that these maps belong to a series of comprehensive territorial plans from 1972-2001. This spatial development is all part of a larger, national plan, which is then taken down to the regional administrative level. At the regional level, the atlas turns to research and development, local economies, industry, demographics, and quality of life. The quality of life illustrations are some of my favorites, along with the maps of population and human settlement. I really appreciated the combination of maps, charts, and photographs in this section of the Atlas, and it was interesting to see the juxtaposition of maps developed from newer GIS technologies with the ancient maps found on pages 24 through 33. I am left with the impression, however, that these newer technologies could have been utilized more fully, such as to intersect layers of data. For example, school data and happiness data could have been combined to show if children attending school in the Republic of Korea are happier than school children elsewhere in the world. Instead the reader has to find and compare data from different places in the Atlas to discover this.

The fourth section is "Korea in the World," and it begins with a world map on Robinson's projection, with countries color-coded by the date of establishment of diplomatic relations, and superimposed with colored dots representing the types of major treaties in effect. This base map is used, with a of couple variants and exceptions, throughout this section to illustrate Korea's place on the world political stage, and in such global economic fields as international investment and trade, global research, and lending/aid to other countries. The last part of this section looks at the cultural richness of Korean heritage through photographs of sites and celebrations, with the location of each mapped. There are photos of temples and historic villages, as well as of dances, martial arts, festivals, and collections of cultural artifacts with historic significance. These include the Baegun Hwasang Chorok Buljo Jikji Simchi Yojeol-roughly translated as the Anthology of the Great Buddhist Priests' Zen Teachings - which was produced in 1377, and is the oldest known book printed with movable metal type anywhere in the world.

The final pages of this atlas contain three beautiful, 1:1,2000,000-scale maps of the Northern, Central, and Southern regions of Korea, and come complete with an Index. All in all, this book can perhaps be best described as it was in the Preface: "the National Atlas of Korea, with name of localities in indigenous language, will circulate a truthful understanding of Korea's physical and human environments internationally" (ii).

\section{OBTAINING THIS ATLAS}

All volumes of The National Atlas of Korea are available online for viewing or download at no cost by visiting nationalatlas.ngii.go.kr.

Distribution of the five hardcover volumes of the atlas has been delegated to the Korean Geographical Society (KGS). We are told that KGS is distributing a very limited number of the books for $\$ 150$ per volume (excluding shipping). It is suggested that interested parties contact KGS directly at this address: Korean Geographical Society. 1413-ho, 213-12. Saechang-ro. Yongsan-gu. Seoul. 140871. Korea.

\section{REFERENCE}

Savada, Andrea Matles, and William Shaw, eds. 1992. A Country Study: South Korea, 4th edition. Washington, DC: Library of Congress. https://www.loc.gov/ item/91039109.

\title{
THE NATIONAL ATLAS OF KOREA, VOLUME III: HUMAN GEOGRAPHY
}

\footnotetext{
Edited by Bae-Gyoon Park, Wonho Lee, THE NATIONAL
ATLAS OF KOREA III

Edited by Bae-Gyoon Park, Wonho Lee,
Chul Sue Hwang, Jinmu Choi, Jongnam Choi, and Gregory Chu

Ministry of Land, Infrastructure, and Transport; and National Geographic Information Institute (NGII), Republic of Korea,2016
}

252 pages. Free online; see "Obtaining this Atlas," below.

ISBN: 978-89-93841-23-7

Review by: Yeong-Hyun Kim, Ohio University 
In 2016, the National Geographic Information Institute (NGII) of the Republic of Korea (South Korea) published the National Atlas of Korea (English Edition), a much-revised third edition of a work first published in 1989. Due to the South Korean Ministry of Land, Infrastructure, and Transport's continued support, the new edition was published in five volumes in print, and is also freely available online. All of the maps, text, tables, and illustrations of the Atlas are available at nationalatlas.ngii.go.kr in both Korean and English, and are downloadable as PDFs. The focus of this review is on Volume III of the National Atlas of Korea, which is dedicated to human geography.

This volume of the Atlas aims to present both the dynamic transformation and the sustainable development of South Korea's "national territory and human life" (page iv). It consists of four sections: the first, "National Territory and Places of Life," provides a geographical overview of South Korea's human-environment interactions, with a particular focus on urban and regional development. The second section, "Population and Living," addresses population growth, distribution, and migration along with their impacts. This section also highlights several important recent changes in South Korea's demographic trends that include an aging population, a small but growing foreign-born population, and a significant increase in single-person households. The third section of the volume, "Industrial Activities," is devoted to the structural and spatial transformation of the South Korean economy, looking at the changing location of economic activities both within South Korea and across international borders, as well as the rise of new industries and growth sites. The fourth and final section, "Society and Culture," describes the sociocultural, political, health, and educational geography of South Korean society. Volume III of the National Atlas of Korea brings together 16 sub-sections on the economic, urban, social, cultural, political, population, and environmental geography of South Korea, with articles contributed by many of that country's best-known and respected human geographers.

The English edition of the Atlas is undoubtedly one of the most comprehensive and valuable sources of information for the general public about contemporary South Korea. For both students and schoolteachers, it should serve as a very useful reference to help them visualize what is happening in that country. Many academics, researchers, and other professionals who are interested in understanding key issues in the changing human geography of South
Korea will also find the Atlas useful in identifying practical data sources and, more generally, providing a starting point for further exploration.

According to its "Preface," Volume III of The National Atlas of Korea should serve to promote "an accurate understanding" of South Korea to future generations of Koreans and achieve "a truthful understanding of Korea's human environments" (page iv) around the world. One may ask whether it is possible, or even desirable, for a national atlas to provide an accurate and truthful account of a country's human geography, something that is subject to diverse and often mutually contradictory interpretations. South Korea's human geography is changing rapidly and somewhat unpredictably with the ongoing economic and geopolitical changes in the East Asian region, and whether this picture of the land and its people will retain its relevance in the future is not at all assured.

The human geography of South Korea has been significantly transformed in recent years, but at the same time it has continued to maintain many of its basic elements. Volume III of The National Atlas of Korea is comprehensive, but it lacks a concluding section which could have offered a much more integrative summation of the various issues and their implications for the land and the people of the Korean Peninsula. It would also have been much more helpful if each section and subsection of the volume had a short introductory overview describing the core themes and issues it covers, and how they are related to those in other sections. It is never easy for an extensively comprehensive atlas, such as The National Atlas of Korea, to present a series of interrelated and connected themes, but clearly written and informative introductions would have helped.

These interconnections are further confused by the organization of some of the subsections within sections. For example, the "Sustainable Land Development" subsection of "National Territory and Places of Life" covers a range of diverse topics, including regional development plans, natural disasters, accidents, and crimes, which might not be particularly relevant to a discussion of sustainable land development. If the goal was to use a human security approach in analyzing the sustainable development of South Korea, this material might better have been combined with the "Use of National Territory" subsection covering challenges in food, water, and energy security, among other topics. Another example of the awkward organization of subsections and topics can be found in the 
"Industrial Activities" section. While international trade has played and continues to play a highly significant role in the growth and expansion of South Korea's industrial activities, this topic is discussed only briefly (150-151) and is not emphasized in the subsections detailing individual manufacturing and service industries.

Despite its shortcomings, Volume III of The National Atlas of Korea: Human Geography is an important contribution to furthering our geographical knowledge and awareness of current issues in South Korea. It is an authoritative source of facts, statistics, and of course maps about South Korea's people, economy, culture, cities, industries, politics, and other human geographical features.

\section{OBTAINING THIS ATLAS}

All volumes of The National Atlas of Korea are available online for viewing or download at no cost by visiting nationalatlas.ngii.go.kr.

Distribution of the five hardcover volumes of the atlas has been delegated to the Korean Geographical Society (KGS). We are told that KGS is distributing a very limited number of the books for $\$ 150$ per volume (excluding shipping). It is suggested that interested parties contact KGS directly at this address: Korean Geographical Society. 1413-ho, 213-12. Saechang-ro. Yongsan-gu. Seoul. 140871. Korea.

\section{GEODESY: INTRODUCTION TO GEODETIC DATUM AND GEODETIC SYSTEMS}

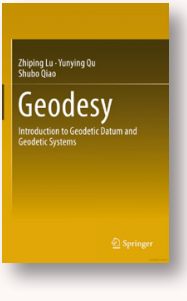

Zhiping Lu, Yunying Qu, and Shubo Qiao

Springer, 2014

401 pages, \$129.99 softcover, \$99 eBook

ISBN: 978-3-642-41244-8

Review by: Fritz Kessler, The Pennsylvania State University

Where is $13^{\circ} 42^{\prime} 56^{\prime \prime}$ and $127^{\circ} 47^{\prime} 33^{\prime \prime}$ located? This seemingly simple question is fraught with complexities. Let's begin by assuming that the latitude value is first and longitude is listed second. Next, one would need to know the cardinal direction (north or south latitude and east or west longitude) associated with each value. If we say that $13^{\circ} 42^{\prime} 56^{\prime \prime}$ is south and $127^{\circ} 47^{\prime} 33^{\prime \prime}$ is east, then this point is located in or near East Grace Park, Caloocan, in The Philippines. However, to precisely and accurately tie these coordinates to a location on the Earth's surface requires a datum. A datum provides the horizontal and vertical control that allows determination of accurate coordinate locations and elevations. Datums are important concepts in the field of geodesy, and are just one of the range of classical and modern themes in that field that are the subject of Geodesy: Introduction to Geodetic Datum and Geodetic Systems. This textbook-the authors of which are on the faculty of People's Liberation Army Information Engineering University in Zhengzhou, Henan Province, People's Republic of China-is an English edition of a text that was originally published in Chinese in 2006.
Geodesy begins with a preface that explains the motivation for pursuing this topic. It is followed by an abbreviations reference section that defines the dozens of acronymsacronyms that can be challenging even to an experienced reader-that appear throughout the book. The table of contents provides a very detailed description of each chapter's contents, and there is also a one-page biographical overview of the authors and their affiliations.

Chapter 1 begins with an introduction to geodesy and its objectives, and then surveys the fields that fall under that rubric. Applications of geodesy in fields such as engineering construction, geosciences, and disaster prevention are discussed in turn, followed by the historical development of different forms of geodesy-physical, satellite, and dynamic. Other topics briefly discussed in the latter sections of the chapter include space geodesy, satellite navigation, and the Earth's gravity field. A short Review and Study Questions section closes this chapter, and is a feature found in all chapters in this book.

Chapter 2, "Geodetic Data Collection Techniques," details the specific measurements, techniques, and instrumentation that can be used to derive data for geodetic purposes. It is divided into two parts, the first of which provides an overview of various measurements that are characteristically collected for geodetic purposes, including horizontal and vertical angles, distances, and astronomic observations such as astronomic latitude, longitude, and azimuth. This part also discusses the equipment used for that collection, and the collection methods employed. Tools of the trade, 